\title{
Simultaneous bilateral laparoscopic nephrectomy in a child with peritoneal catheter dialysis using a 4-port trans-abdominal technique
}

\author{
Claudio De Carli, MD; Luis A. Guerra, MD
}

Children's Hospital of Eastern Ontario, Ottawa, ON

Cite as: Can Urol Assoc J 2015;9(1-2):59-61. http://dx.doi.org/10.5489/cuaj.2465

Published online February 5, 2015.

\section{Abstract}

We present the case of an 11-year-old girl with end-stage renal disease and a previously-inserted peritoneal dialysis catheter who underwent a bilateral transperitoneal laparoscopic nephrectomy for hypertension refractory to medical treatment. We employed a 4-port transperitoneal technique using the first detached kidney to occlude the ipsilateral abdominal wall access port during the contralateral nephrectomy to avoid gas/fluid leak and to facilitate location of the first kidney at the end of the surgery. The patient had no morbidity and was able to resume use of the peritoneal dialysis catheter 5 days after the surgery.

\section{Introduction}

Transperitoneal bilateral laparoscopic nephrectomy (BLN) for end-stage renal disease in children has been welldescribed..$^{1-3}$ It remains a challenging surgery due to the necessity of multiple access ports and the need to move the patient to an opposite decubitus flank position after the first nephrectomy. Despite closing the incision, the initial access port is prone to leakage of fluid or gas during the contralateral nephrectomy. In BLN, it may also be difficult to locate the first kidney - which is usually loose in the cavity - for removal at the end of surgery. Further complicating the procedure is the fact that some patients have a previously inserted peritoneal dialysis catheter (PDC) in place.

We describe the case of a young girl with a PDC who underwent transperitoneal BLN employing a minimum number of ports, and using the first kidney to occlude the inferior iliac port during the nephrectomy on the contralateral side.

\section{Case report}

An 11-year-old girl presented with acute renal failure progressing to end-stage renal disease. She was previously healthy with no related family history, no urinary symptoms or previous elevated creatinine and urea. The etiology of her kidney disease was not known and the only positive finding from her extensive workup was DNA for parvovirus B19 in her renal biopsy, which also demonstrated diffuse scarring but no inflammation. Her body mass index was 16.9. She was started on hemodialysis and initially progressed well; however, her systolic blood pressure (SBP) steadily increased to levels above $180 \mathrm{mmHg}$ and became refractory to multiple hypertensive medications. The patient subsequently developed seizures, and intracranial bleeding with intraparenchymal temporal-parietal hemorrhage.

Given the persistent elevated SBP, the patient was switched from hemodialysis to peritoneal dialysis and a PDC was placed laparoscopically. Despite administration of multiple anti-hypertensive medications, she continued to experience SBP peaks of about $180 \mathrm{mmHg}$ along with headaches and shortness of breath.

\section{Surgery}

The patient underwent successful transperitoneal BLN in February 2009 using monopolar coagulators with no complications. The operating time was 240 minutes. Blood loss was minimal (50-100 mL) and the patient did not require transfusions. She was able to start on a normal diet the following day, peritoneal dialysis was restarted 5 days later, and the patient was discharged 7 days after surgery. Time to initiation of dialysis and length of stay were extended as precautions due to this patient's history of severe hypertension and associated intra-cerebral hemorrhage.

After several weeks the patient's symptoms had improved and she was weaned to a maintenance dose of anti-hyper- 
tensive medication; 6 months later she was able to stop all anti-hypertensive medications. She was followed monthly by nephrology until she received a cadaveric renal transplant 21 months after the nephrectomies.

\section{Surgical technique}

The patient was positioned on the operating table on her left side, at a $45^{\circ}$ angle. Following the right nephrectomy, she was re-repositioned to perform the contralateral nephrectomy. A $5-\mathrm{mm}, 30^{\circ}$ scope was inserted in the umbilicus using the Hassan technique. Pneumoperitoneum was performed at $10 \mathrm{mmHg}$ pressure. The epigastric port was used as a working port for both hemi-abdomens. Two additional 5 -mm ports were inserted in each iliac fossa. All ports were functional and no additional ports were needed (Fig. 1).

Starting with the right side, the large bowel was retracted medially. The right ureter was identified and dissected as low as possible, clipped, and divided. The proximal end of the ureter was used for retraction to facilitate the dissection and isolation of the renal hilum. The renal vessels were also clipped and divided. Cauterization of the remaining peritoneal attachments completed the kidney dissection. Instead of leaving the right kidney resting in situ or in a predetermined location, we pulled the right ureter through the more lateral iliac port, and then removed the trocar, leaving the ureter externalized and held in place by a hemostat with the kidney positioned firmly against the abdominal wall, and a sterile dressing covering the opening (Fig. 2). The same nephrec-

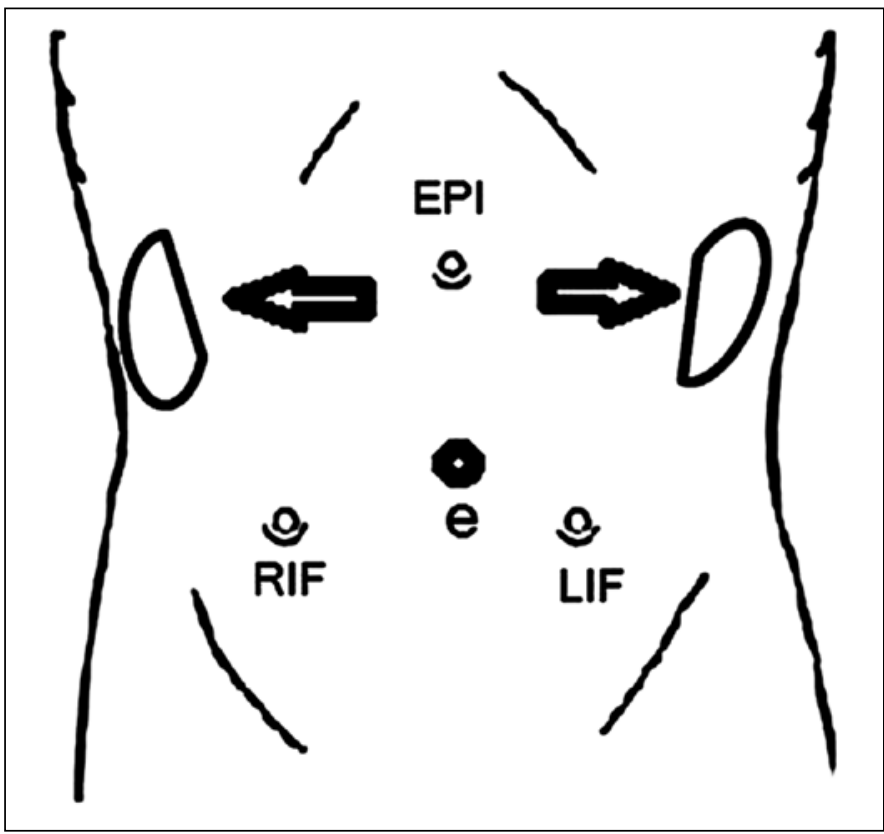

Fig. 1. Surgical technique. e: endoscope; EPI: epigastric port (arrows show that a single port can be used for both nephrectomies); RIF: right iliac fossa; LIF: left iliac fossa. tomy technique was used on the left side. Both kidneys were then removed through a $5-\mathrm{cm}$ transverse incision from an extension of the left access port. All abdominal wall incisions were closed using standard procedure. A postoperative drain was not deemed necessary.

\section{Discussion}

Pediatric bilateral nephrectomy may be required in selected patients and prior to renal transplantation for end-stage renal disease; it is quite a safe procedure from a surgical and anesthetic perspective, with good outcomes. ${ }^{1,4}$ Laparoscopic nephrectomy has advantages compared to open surgery, ${ }^{5}$ and either a transperitoneal or retroperitoneal approach may be used. ${ }^{6}$

The retroperitoneal approach has 2 main advantages: avoiding repositioning of the patient and preserving the peritoneum to allow continuation of peritoneal dialysis. This technique is particularly useful for small kidneys, but it offers a small working space. The transperitoneal approach offers more space to maneuver; however, there is a possibility of fluid or gas leakage from the ports used for the first nephrectomy when the patient is repositioned, and the resumption of peritoneal dialysis may be delayed.

Despite a previous report of BLN using 3 ports in an infant, ${ }^{7}$ the older and adolescent patient usually demands a more challenging dissection and surgical exposure. Our

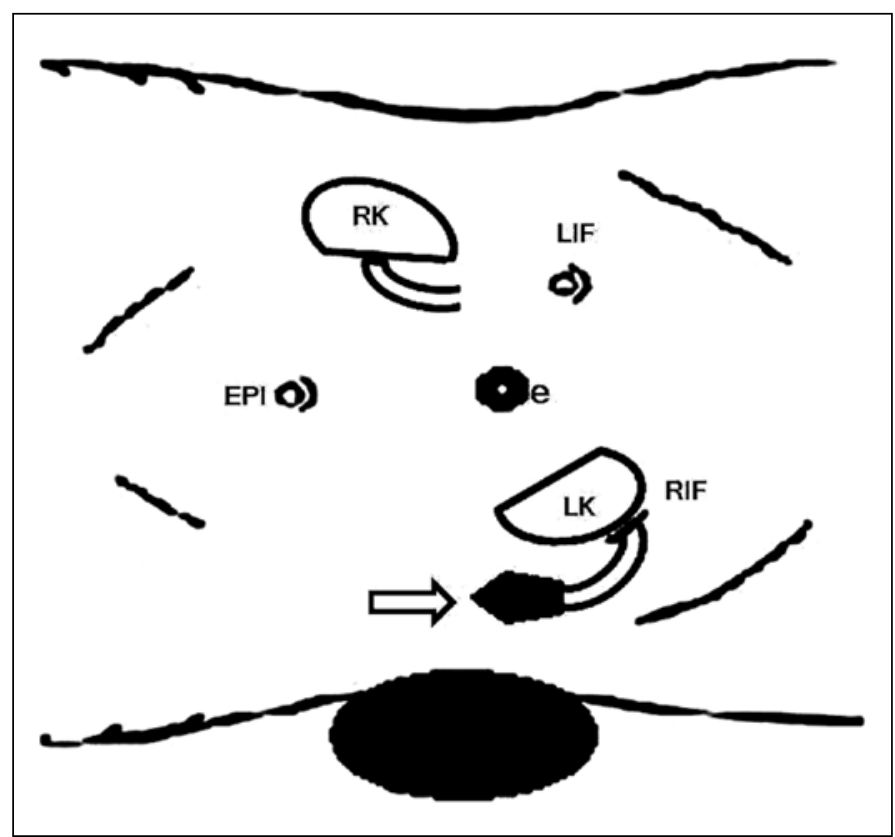

Fig. 2. Patient position and exteriorization of the first removed kidney for the second nephrectomy. The RK is attached to the abdominal wall by externalizing its ureter through the RIF port, the ureter is held against the skin by an hemostat and covered by a Tegaderm sterile dressing (arrow). RK: right kidney; LK: left kidney; e: endoscope; EPI: epigastric port; RIF: right iliac fossa; LIF: left iliac fossa. 
Simultaneous bilateral laparoscopic nephrectomy in a child

technique overcomes some of the possible disadvantages of the transperitoneal approach for simultaneous BLN. First, by using the minimum number of ports, recovery time may be shortened so that peritoneal dialysis may be resumed sooner. The 4-port technique allowed for safe and quick completion of the bilateral procedures with the epigastric and umbilical ports used effectively for both nephrectomies. The surgical time in this case was significantly shorter than other reported bilateral synchronous nephrectomies, although in some of these cases additional procedures were done simultaneously (catheter insertion and hernia repair). ${ }^{8}$ Time to successful restart of peritoneal dialysis of 5 days was relatively short compared to the 10 to 14 days reported elsewhere, ${ }^{9,10}$ although it should be noted that the retroperitoneal approach appears to allow dialysis to be restarted sooner. ${ }^{8}$ In addition, the single incision/port technique in children reduces the risk of wound infection and subcutaneous emphysema while improving cosmetic outcomes. ${ }^{11,12}$ The advantage may be limited, however, in cases of simultaneous bilateral nephrectomy since 2 incisions (one for each nephrectomy) are needed. Furthermore, one needs to consider that the single incision is likely to be longer than any one incision in a multi-port technique to accommodate the single-port device, reducing the cosmetic benefit. Finally, fixing the first kidney against the ipsilateral access port while performing the contralateral nephrectomy provided 2 advantages: blocking the port with the kidney prevented fluid and gas leakage while allowing the surgeon to locate the kidney more easily for removal at the end of the surgery, possibly shortening the operating time.

Our technique is an option that surgeons may consider when selecting a simultaneous BLN approach. There are many factors that must be weighed, including patient and kidney size and the surgeon's personal experience; currently there is no conclusively optimal approach for the performance of BLN.

\section{Conclusion}

The transperitoneal, 4-port BLN is a safe and feasible technique. Occluding the inferior abdominal port with the ipsilateral kidney, while performing the second nephrectomy, may prevent fluid and gas leakage while reducing the operating time.
Acknowledgments: The authors thank Carolyn Wayne and Jennifer Vincent for their help in preparing this manuscript.

Competing interests: Authors declare no competing financial or personal interests.

This paper has been peer-reviewed.

\section{References}

1. Shiro YN, Bruce SH, Luay SP, et al. Mortality and morbidity in pretransplant bilateral nephrectomy. J Urol 1978;12:55-8. http://dx.doi.org/10.1016/0090-4295(78)90367-9

2. Shoma AM, Erakey I, E-Kappany HA. Pretransplant native nephrectomy in patients with end-stage renal failure: Assessment of the role of laparoscopy. J Urol 2003;61:915-20. http://dx.doi.org/10.1016/ S0090-4295(02)02556-6

3. Tanaka K, Kawabata G, Takeda M, et al. Posterior approach for retroperitoneal laparoscopic bilateral native nephrectomy in prone position: Initial experience with four cases. Int Jour Urol 2007;14:975-7. http://dx.doi.org/10.1111/i.1442-2042.2007.01864.x

4. Modi MP, Vora KS, Parikh GP, et al. Anaesthetic consideration during laparoscopic bilateral simultaneous nephrectomy. J Minim Access Surg 2014;10:10-3. http://dx.doi.org/10.4103/0972-9941.124453

5. Wagner $S$, Greco F, Doehn C, et al. Laparoscopic versus open bilateral nephrectomy in transplant recipients with medication-resistant hypertension: Final results of a multicenter study with 15 years of follow-up. Transplant Proc 2011;43:2557-61. http://dx.doi.org/10.1016/j.transproceed.2011.04.011

6. Kim C, McKay K, Docimo SG. Laparoscopic nephrectomy in children: Systematic review of transperitoneal and retroperitoneal approaches. J Urol 2009;73:280-4. http://dx.doi.org/10.1016/i.urology.2008.08.471

7. Zuniga $Z V$, Ellis D, Mortiz ML, et al. Bilateral laparoscopic transperitoneal nephrectomy with earlyperitoneal dialysis in an infant with the nephrotic syndrome. J Urol 2003;170:1962. http://dx.doi. org/10.1097/01.ju.0000092311.14936.8a

8. Szymanski KM, Bitzan M, Capolicchio JP. Is retroperitoneoscopy the gold standard for endoscopic nephrectomy in children on peritoneal dialysis? J Urol 2010;184(4 Suppl):1631-7. http://dx.doi. org/10.1016/i.juro.2010.04.019

9. Malavade TS, Bargman JM. The outcome of nephrectomy in peritoneal dialysis patients. Adv Perit Dial 2013;29:25-8.

10. Rais-Bahrami $S$, Romeroemail $F R$, Lima $G C$, et al. Reinstatement of continuous ambulatory peritoneal dialysis after transperitoneal laparoscopic nephrectomy. Urology 2006;68:715-7. http://dx.doi. org/10.1016/i.urology.2006.04.032

11. Cherian A, De Win G. Single incision retro-peritoneoscopic paediatric nephrectomy: Early experience. J Pediatr Urol 2014;10:564-6. http://dx.doi.org/10.1016/i.ppurol.2013.12.018

12. Johnson KC, Cha DY, DaJusta DG, et al. Pediatric single-port-access nephrectomy for a multicystic, dysplastic kidney. J Pediatr Urol 2009;5:402-4. http://dx.doi.org/10.1016/i.jpurol.2009.03.011

Correspondence: Dr. Luis A Guerra, Children's Hospital of Eastern Ontario, Ottawa, ON; lguerra@cheo.on.ca 